\title{
Dietary intervention rescues maternal obesity induced behavior deficits and neuroinflammation in offspring
}

\author{
Silvia S Kang ${ }^{1}$, Aishe Kurti ${ }^{1}$, Damien A Fair ${ }^{3,4}$ and John D Fryer ${ }^{1,2^{*}}$
}

\begin{abstract}
Obesity induces a low-grade inflammatory state and has been associated with behavioral and cognitive alterations. Importantly, maternal environmental insults can adversely impact subsequent offspring behavior and have been linked with neurodevelopmental disorders such as autism spectrum disorder (ASD) and attention deficit hyperactivity disorder $(A H D H)$. It is unknown if maternal obesity significantly alters offspring sociability, a key ASD feature, and if altering maternal diet will provide an efficacious intervention paradigm for behavioral deficits. Here we investigated the impact of maternal high fat diet (HFD) and maternal dietary intervention during lactation on offspring behavior and brain inflammation in mice. We found that maternal HFD increased anxiety and decreased sociability in female offspring. Additionally, female offspring from HFD-fed dams also exhibited increased brain IL-1 $\beta$ and TNFa and microglial activation. Importantly, maternal dietary intervention during lactation was sufficient to alleviate social deficits and brain inflammation. Maternal obesity during gestation alone was sufficient to increase hyperactivity in male offspring, a phenotype that was not ameliorated by dietary intervention. These data suggest that maternal HFD acts as a prenatal/ perinatal insult that significantly impacts offspring behavior and inflammation and that dietary intervention during lactation may be an easily translatable, efficacious intervention to offset some of these manifestations.
\end{abstract}

Keywords: Maternal obesity, Dietary intervention, Neuroinflammation, Behavior, ASD, ADHD

\section{Background}

Maternal obesity is a pervasive health issue, with over $30 \%$ of child-bearing age women in the United States being categorized as obese [1]. Adverse symptoms such as gestational diabetes, pre-term birth, and birth injury are associated with maternal obesity [2-4]. Emerging data have also linked maternal obesity with long-lasting ramifications in offspring including altered immunity, metabolism, increased obesity, and cardiovascular risk [5-11]. Maternal obesity-induced changes in offspring brain lipid peroxidation, hippocampal neurogenesis, and inflammation [12-14] may also impact cognition and behavior.

Obesity is associated with increased risk of behavioral disorders. In humans, increased body mass index (BMI) has been connected with elevated anxiety and depression

\footnotetext{
* Correspondence: fryer.john@mayo.edu

'Department of Neuroscience, Mayo Clinic Jacksonville, 4500 San Pablo Road, Jacksonville, FL 32224, USA

${ }^{2}$ Neurobiology of Disease Program, Mayo Graduate School, 200 First Street SW, Rochester, MN 55905, USA

Full list of author information is available at the end of the article
}

[15-18]. Interestingly, maternal obesity also increased anxiety in non-human primate and rodent offspring [14,19-21]. Altered maternal environments are implicated in neurodevelopmental disorders such as attention deficit hyperactivity disorder (ADHD) and autism spectrum disorder (ASD). ADHD symptoms in children have been associated with pre-pregnancy adiposity [22-24] and animal models of maternal high fat diet (HFD) revealed increased offspring activity [25]. Furthermore, children from mothers with a pre-pregnancy body mass index $\geq 35.0$ exhibited delayed mental development as well as learning and behavioral disabilities [26,27]. In humans, maternal obesity or infection has also been linked to increased autism risk in children [28-30]. While rodent modeling of maternal infection results in offspring displaying features of ASD [31,32], it is unknown whether maternal obesity alone induces any ASD-like behaviors in subsequent offspring.

Growing evidence suggests a link between altered immunity and aberrant behavior and cognition [31,33,34]. 
Pro-inflammatory cytokines, including interleukin (IL)- $1 \beta$ and tumor necrosis factor (TNF) $\alpha$, have been associated with increased anxiety, altered cognitive function [35-38], and are elevated in ASD patients [39-41]. Activation of microglia, the central nervous system (CNS) resident immune cells, often coincides with behavioral manifestations [14,42-45]. Although maternal obesity has been suggested to increase brain inflammation [14], intervention strategies that may alleviate maladaptive behaviors and CNS inflammation observed in offspring have not been explored.

Human data suggest that maternal obesity may be linked to ASD-like behaviors [30]; however, to date, little is known whether rodent models of maternal obesity will recapitulate any aspect of ASD in offspring and provide a model to examine the efficacy of intervention strategies. Additionally, it is unclear whether maternal intervention strategies would prove to be efficacious against neuroinflammation that is observed in offspring as a result of maternal HFD [14] in addition to any behavioral abnormalities. Here we investigated the effects of maternal obesity and maternal dietary intervention during lactation on offspring in a rodent model. Specifically, offspring behavior from HFD-fed dams without dietary intervention was assessed for behavioral abnormalities including anxiety, hyperactivity and sociability, as well as neuroinflammation. These data indicate that maternal obesity impacts several aspects of behavior, including sociability, and evokes neuroinflammation in offspring. Importantly, modulation of offspring behavior and neuroinflammation by maternal dietary intervention suggests that a significant alleviation of offspring abnormalities can be achieved using an easily translatable intervention strategy.

\section{Materials and methods}

\section{Animals}

C57BL/6 J mice (The Jackson Laboratory, Bar Harbor, ME, USA) were housed under standard laboratory conditions in ventilated cages on 12-hour light:dark cycles in a specific pathogen-free environment. Animal protocols were reviewed and approved by Mayo Clinic Institutional Animal Care and Use Committee.

\section{Diets and litters}

Six-week-old female breeders were placed on either control diet (CD) (10\% of calories from fat; Research Diets, New Brunswick, NJ, USA \#D12450B) or HFD (60\% of calories from fat; Research Diets New Brunswick, NJ, USA \#D12492). Comparison of the diets is shown in Additional file 1: Table S1. Mice were fed ad libitum for six weeks prior to breeding with a two- to three-monthold male. Females were either maintained on the gestation diet or on post-natal day 0 were switched to the opposing diet for the duration of lactation. This created four diet conditions: CD/CD, HFD/HFD, HFD/CD and CD/HFD, indicating gestation/lactation diets respectively. To reduce the impact of litter effects, litters were adjusted to no more than nine per dam. The average litter sizes for the $\mathrm{CD} / \mathrm{CD}=5.2, \mathrm{HFD} / \mathrm{HFD}=4.2, \mathrm{CD} / \mathrm{HFD}=8.3$ and $\mathrm{HFD} /$ $\mathrm{CD}=4$. The only significant difference was between $\mathrm{CD} /$ HFD versus HFD/HFD $(P=0.02)$ and HFD/CD $(P=0.03)$ using a one-way analysis of variance (ANOVA) with Tukey post-hoc testing. Litter size did not appear to impact subsequent pup weight or behavior. For example, linear regression analysis of litter size versus interaction score, an important parameter in the three-chamber social interaction assay, revealed no significant correlation between litter size and behavior (linear regression $\mathrm{R}^{2}=$ $0.02557, P=0.177$ ). Additionally, no associations were observed between litter size and pup weight (Additional file 2: Figure $\mathrm{S} 1$ ). The total number of dams used was $\mathrm{CD} / \mathrm{CD}=$ $11, \mathrm{HFD} / \mathrm{HFD}=8, \mathrm{CD} / \mathrm{HFD}=4, \mathrm{HFD} / \mathrm{CD}=5$. For behavior, the number of male and female offspring used is presented in the graph. A subset was randomly selected for biochemistry. All offspring were weaned at postnatal day (P)21 and were placed on irradiated standard chow (Harlan Laboratories; Madison, WI, USA).

\section{Behavioral testing}

Behavior was assessed between 9:00 am and 5:00 pm. For all behavioral tests, mice were acclimated in the room for an hour prior to the onset of testing. The apparatus was thoroughly cleaned with $30 \%$ ethanol between test mice to remove any residual odors in the equipment. For all behavioral tests, data were recorded and monitored using Fujinon cameras mounted overhead in conjunction with Anymaze tracking software (Stoelting Co.; Wood Dale, IL, USA). Specific regions of interest were defined digitally using Anymaze software for each test and the center body of the animals was subsequently tracked for the duration of the test in order to calculate behavioral measurements from the recorded visual files. Raw data produced by Anymaze software were then analyzed as described in section data analysis and statistics.

\section{Open field assay}

Mice were placed in a $40 \times 40 \mathrm{~cm}$ black Plexiglas box with a brightly lit center. Behavioral activity was recorded by Fujinon cameras for 15 minutes and was tracked using Anymaze software (Stoelting Co.; Wood Dale, IL, USA). An imaginary $13 \mathrm{~cm} \times 13 \mathrm{~cm}$ region in the center of the box and a perimeter region were defined using Anymaze software. Center:total distance ratios were computed by Anymaze software by taking the total distance traveled in the pre-defined center region relative to the total distance traveled during the 15 minute observation period. Side mounted photobeams located $7.6 \mathrm{~cm}$ from the floor of the box were used to monitor rearing whereas other measures 
(for example. total distance traveled and center:total distance) were acquired using the overhead camera.

\section{Three-chamber social interaction test}

Social behavior was tested in a 3-chamber Plexiglas $40 \times 40$ $\mathrm{cm}$ box consisting of two $17 \times 40 \mathrm{~cm}$ regions separated by 2 dividers forming a smaller $5 \times 40 \mathrm{~cm}$ center region. Mice were able to move freely through a small $8 \times 5 \mathrm{~cm}$ opening that was aligned in both dividers. Each of the larger chambers contained an inverted wire mesh cylinder in opposing corners. Mice were initially acclimated to the box and empty cylinders for four minutes and then placed in temporary holding cages. A sex-matched probe mouse of the same strain was placed in one of the inverted mesh cylinders and the test mouse was placed back in the box. The open mesh enabled visual, olfactory, and auditory interactions between probe and test mice. Test mice were subsequently monitored for ten minutes in the presence of the probe mouse using Fujinon cameras and Anymaze software (Stoelting Co.; Wood Dale, IL, USA). Imaginary circular regions encompassing the empty cylinder and the probe mouse cylinder were defined using Anymaze software. Time in mouse cup minus time in empty cup region was then calculated using these pre-defined regions. Interaction scores, which normalize for the total time spent in both regions, were calculated by the following formula:

$\left(\right.$ Time $_{\text {Mouse cup }}-$ Time $\left._{\text {empty cup }}\right) /\left(\right.$ Time $_{\text {Mouse cup }}+$ Time $\left._{\text {empty cup }}\right)$

\section{Weight and general procedures}

Female mice were weighed prior to the onset of diet initiation and then on a weekly basis prior to and through gestation. Offspring were weighed at weaning (P21) and prior to commencement of behavioral testing. For biochemistry and immunohistology, a subset of animals was harvested 24 hours after the final behavior test.

\section{Tissue processing}

Animals were deeply anesthetized with pentobarbital prior to cardiac perfusion with PBS to expunge blood from the cerebrovasculature. For biochemical analysis, hemi-brain tissues were quickly frozen on dry ice until further processing. Tissues were briefly sonicated in Tris buffered saline with EDTA (TBSE) (50 mM Tris $\mathrm{pH}=7.5,150 \mathrm{mM} \mathrm{NaCl}$, $1 \mathrm{mM}$ EDTA) with $1 \mathrm{X}$ protease and phosphatase inhibitors (Thermo Scientific, Waltham, MA, USA). An aliquot of this sonicated tissue suspension was immediately placed into Trizol LS for RNA isolation using the Direct-zol RNA kit (Zymo Research, Irvine, CA, USA). Another aliquot was centrifuged for 15 minutes at $20,000 \times g$ at $4^{\circ} \mathrm{C}$ and the soluble TBSE fraction was isolated for cytokine assessment. TBSE tissue protein levels were assessed using a BCA kit (Thermo Scientific, Waltham, MA, USA).

\section{Immunohistochemistry}

Brains obtained from animals perfused with PBS followed by $10 \%$ normal buffered formalin (NBF) were further dropfixed overnight in $10 \% \mathrm{NBF}$ at $4^{\circ} \mathrm{C}$. Samples were then switched to $30 \%$ sucrose in PBS and incubated overnight at $4^{\circ} \mathrm{C}$. Fifty micron sagittal brain sections were cut on a freezing-sliding microtome and stored in cryoprotectant at $-20^{\circ} \mathrm{C}$ until staining. Tissues were placed in netwells in a 12-well plate and washed with PBS to remove cryoprotectant. Sections were blocked for endogenous peroxidase activity and permeabilized with $0.6 \% \mathrm{H}_{2} \mathrm{O}_{2}, 0.1 \% \mathrm{NaN}_{3}$ in PBS-X (1X PBS containing 0.3\% Triton-X) for 30 minutes at room temperature (RT). Samples were washed $\mathrm{x} 3$ with PBS-X for 10 minutes/wash prior to blocking with $1 \%$ milk PBS-X for 90 minutes at RT. Sections were incubated with 1:5,000 Iba1 (catalog \# 019-9741, Wako, Richmond, VA, USA) in $0.5 \%$ milk PBS-X for 2 days rocking at $4^{\circ} \mathrm{C}$. After $\times 4$ washes with PBS-X at RT, sections were incubated with the Vectastain kit anti-Rabbit IgG component (Vector Labs, Burlingame, CA, USA) for 2 days, rocking at $4^{\circ} \mathrm{C}$. Samples were washed $\times 4$ with PBS-X at RT and incubated with the ABC component for three hours. Iba1 staining was then developed using the DAB kit (Vector Labs, Burlingame, CA, USA) according to manufacturer's instructions. Images were acquired using an Aperio XT Scanner (Aperio, Vista, CA, USA) at a ×20 magnification. Densitometric analysis of Iba1 staining intensity in the amygdala was performed with Image J software.

\section{ELISA}

Tissue cytokine levels were measured from hemi-brain tissue using IL-1 $\beta$ and TNF $\alpha$ enzymelinked immunosorbent assay (ELISA) kits according to manufacturer's instructions (Biolegend, San Diego, CA, USA), with values expressed as $\mathrm{pg} / \mathrm{mg}$ of total protein.

\section{RT-qPCR}

Total RNA was isolated from sonicated tissues using a Direct-zol total RNA isolation kit (Zymo Research, Irvine, CA, USA) according to manufacturer's instructions with in-column DNase I treatment. RNA was reverse transcribed in first strand buffer containing $0.01 \mathrm{M}$ dithiothreitol (DTT), $200 \mathrm{ng}$ random hexamers, $0.5 \mathrm{mM}$ dNTPs and 40U M-MLV according to M-MLV RT protocols (Invitrogen-Life Technologies, Grand Island, NY, USA). To conduct real time quantitative polymerase chain reaction (RT-qPCR), cDNA was added to a reaction mix $(10 \mu \mathrm{L}$ final volume) containing $300 \mathrm{nM}$ gene-specific primers and Universal SYBR green supermix (Bio-Rad, Hercules, CA, USA). All samples were run in triplicate and were analyzed on an ABI 7900 HT Fast Real Time PCR instrument (Applied Biosystems-Life Technologies, Carlsbad, CA, USA) for quantitative monitoring of PCR product formation. Relative gene expression was normalized to Gapdh controls and assessed using the $2^{-\Delta \Delta C T}$ method. Primer 
sequences are as follows: Gapdh: F: CTGCACCACCAAC TGCTTAG; Gapdh: R: ACAGTCTTCTGGGTGGCA GT: Iba1: F: GGATTTGCAGGGAGGAAAAG; Iba1: R: TGGG ATCATCGAGGAATTG.

\section{Data analysis and statistics}

Data were analyzed using Prism statistical software (La Jolla, CA, USA). For maternal weight gain, data are analyzed as a repeated measures ANOVA. Behavioral data were assessed within each sex by one-way ANOVA tests using a Fisher's LSD post-hoc analysis. Biochemical data were examined using one-way ANOVA with Tukey post-hoc analysis. All data with $P<0.05$ were considered significant.

\section{Results}

Maternal high fat diet increases weight in both dams and offspring

To examine the contribution of maternal HFD during gestation and/or lactation on subsequent offspring, female C57BL/6 J mice were placed on either a HFD $(60 \%$ calories from fat) or control diet CD (10\% calories from fat). Diet was initiated six weeks prior to breeding and continued throughout gestation. The average days between initial mating and pup birth was similar for both $\mathrm{CD}$ and HFD-fed dams (mean \pm SEM 24.93 $\pm 1.3 \mathrm{CD}$ versus $25.35 \pm 1.6 ; P=0.8439$, two-tailed $t$-test). At birth, dams were either maintained on the same diet or were switched to the opposing diet, generating four groups presented as gestation diet/lactation diet: $C D / C D$, HFD/HFD, CD/HFD and HFD/CD (Figure 1A). At P21, pups were weaned and placed on standard chow to limit the impact of HFD to the stages associated with maternal care. Dams fed HFD weighed significantly more than CD dams beginning as early as one week after diet initiation and lasting through the last point measured during gestation (Figure 1B). HFD/HFD male and female offspring were significantly heavier at weaning relative to all other conditions within their respective sex (Figure 1C). By P32-35, HFD/HFD male offspring remained significantly heavier than $\mathrm{CD} / \mathrm{CD}$ and $\mathrm{CD} / \mathrm{HFD}$ pups but HFD/HFD female offspring were only significantly heavier than $\mathrm{CD} / \mathrm{CD}$ controls (Figure 1D). Interestingly, at P32-35 both male and female offspring from dams fed HFD during gestation were significantly heavier than $\mathrm{CD} / \mathrm{CD}$ controls regardless of lactation diet. Examination of overall litter size versus female or male weight revealed no significant correlation between these two factors (Additional file 2: Figure S1). These data demonstrate that maternal HFD significantly impacts maternal and offspring weight during the late juvenile stages.
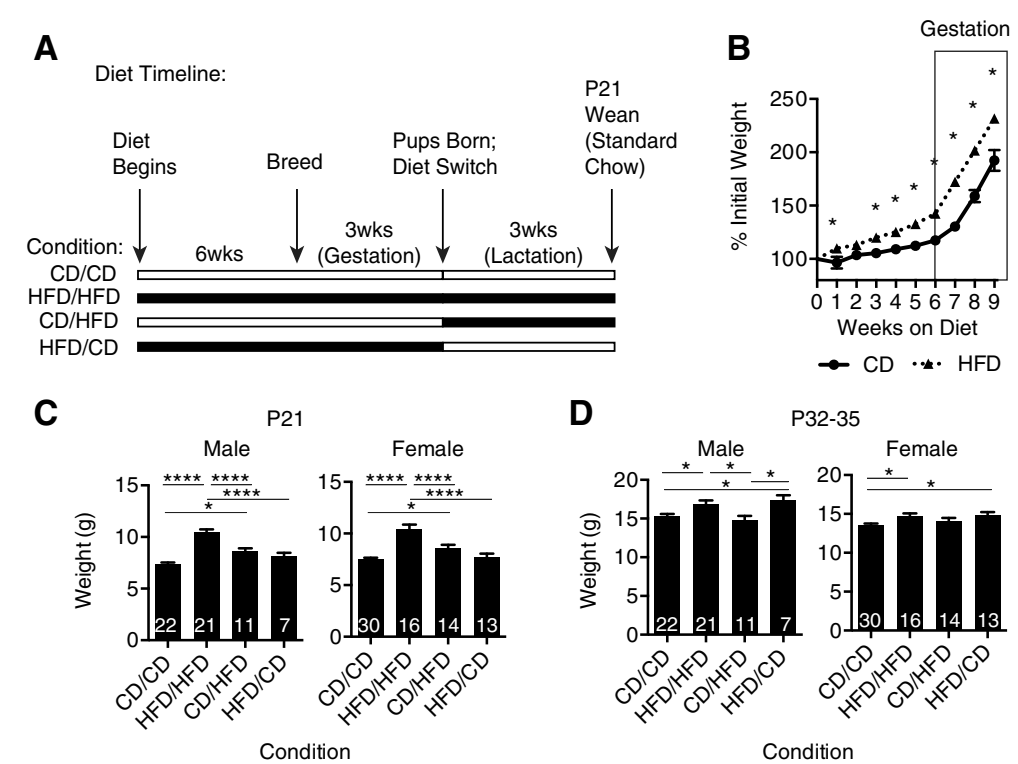

Figure 1 High fat diet (HFD) significantly increases maternal and offspring weight. (A), Timeline for feeding schedule of HFD (60\% calories from fat; black line) or control diet (CD) (10\% calories from fat; white line) for the experimental conditions. (B) Percent initial weight for dams fed CD (black line, circle) or HFD (black dotted line, triangle) 6 weeks prior to and during gestation represented as mean \pm SEM. $N=12$ to 14 per group, ${ }^{*} P<0.01$ (weeks 1 to 3), ${ }^{*} P<0.001$ (week 4) or ${ }^{*} P<0.0001$ (weeks 5 to 9), repeated measures ANOVA. (C-D) Mean weight \pm SEM for male and female offspring from CD/CD, HFD/HFD, CD/HFD or HFD/CD dams at (C), P21 and (D), P32-35 ( $N=7$ to 22 or $N=13$ to 30 per condition for males and females, respectively; one-way ANOVA with post-hoc Tukey analysis; $P 21$ males ${ }^{*} P=0.0156,{ }^{* * * *} P<0.0001 ; P 21$ females ${ }^{*} P=0.045,{ }^{* * * *} P<0.0001 ; P 32-35$ males $\mathrm{CD} / \mathrm{CD}$ versus $\mathrm{HFD} / \mathrm{HFD} * P=0.0169, \mathrm{CD} / \mathrm{CD}$ versus HFD/CD $* P=0.0319, \mathrm{HFD} / \mathrm{HFD}$ versus $\mathrm{CD} / \mathrm{HFD} * P=0.0113, \mathrm{CD} / \mathrm{HFD}$ versus HFD/CD $* P=0.0166$; P32-35 females $C D / C D$ versus HFD/HFD * $P=0.0189, C D / C D$ versus HFD/CD * $P=0.0127)$. Abbreviations: $P$, postnatal day. 
Maternal high fat diet during gestation results in increased anxiety in female offspring and hyperactivity in male offspring

Maternal HFD maintained throughout gestation and lactation has been associated with increased anxiety in subsequent offspring [14,20,21]. To determine if the gestation and/or lactation periods of maternal diet contributed to increased anxiety, offspring from CD/CD, HFD/HFD, CD/ HFD and HFD/CD dams were analyzed in the open field assay (OFA) at P32-35. Maternal diet resulted in a significant increase in distance traveled for male HFD/HFD or $\mathrm{HFD} / \mathrm{CD}$ versus male $\mathrm{CD} / \mathrm{CD}$ offspring (Figure $2 \mathrm{~A}$ ). A trend for increased activity in female HFD/HFD offspring was also observed. Female offspring from HFD/HFD and $\mathrm{HFD} / \mathrm{CD}$ dams relative to $\mathrm{CD} / \mathrm{CD}$ controls demonstrated a significant decrease in the center:total distance ratio, indicative of increased anxiety, suggesting that the gestational period was critical for altering this behavior (Figure 2B). Increased rearing was also observed within both male and female offspring from HFD/HFD dams (Figure 2C), a sign of increased motility and anxiety. Notably, in female offspring, a striking reduction in rearing behavior was observed upon diet switch at lactation (HFD/HFD versus CD/CD, HFD/HFD versus CD/HFD, HFD/HFD versus HFD/CD), suggesting a partial amelioration of some anxiety measures with maternal dietary intervention (Figure 2C).

\section{Maternal diet intervention during lactation alleviates social defects in female offspring}

In models of maternal immune activation that induce inflammation, ASD-like behaviors such as decreased sociability have been observed $[32,46]$. As obesity represents a state of low-grade inflammation, we next determined whether maternal obesity impacted sociability and if dietary intervention during the lactation period of maternal diet could alleviate any anomalies in this behavior.

At 5.5 to 6 weeks of age, offspring from each group were tested for social interactions using the 3-chamber social test. Alterations in sociability were observed in female offspring, but not male offspring, with HFD/HFD offspring showing a significant reduction in interaction time with cups containing probe mice relative to the empty cups in the $\mathrm{CD} / \mathrm{CD}$ group (Figure $3 \mathrm{~A}$ ). When normalized for total time spent in both regions, represented as an interaction score, the same significant decrease was observed in HFD/ HFD female offspring compared to $\mathrm{CD} / \mathrm{CD}$ female offspring (Figure 3B). Importantly, HFD/CD dams that received dietary intervention at the lactation stage yielded offspring that were similar in sociability compared to $\mathrm{CD} /$ CD dam offspring (Figure 3B). Together, these data indicate that maternal diet impacts offspring sociability and dietary intervention during lactation is sufficient to ameliorate this ASD-like phenotype.

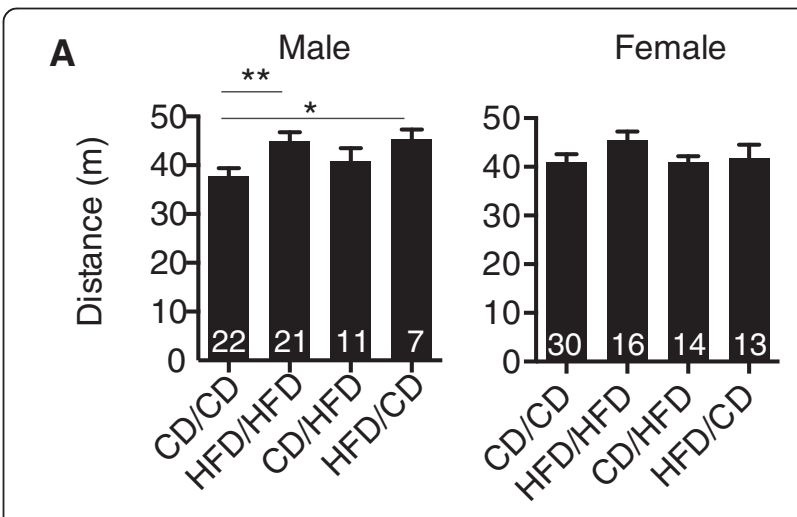

B
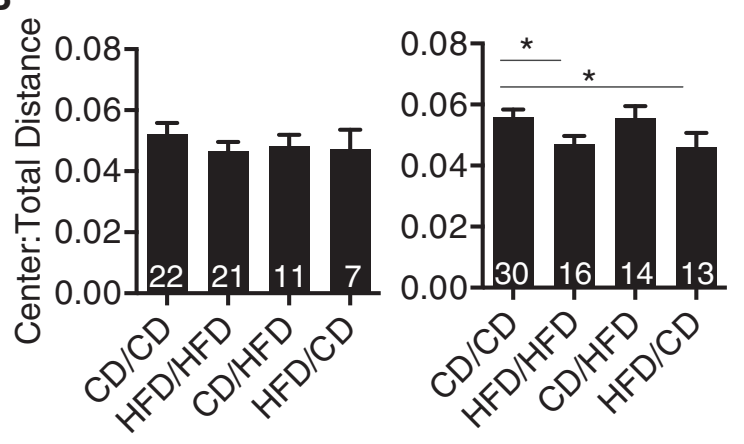

C
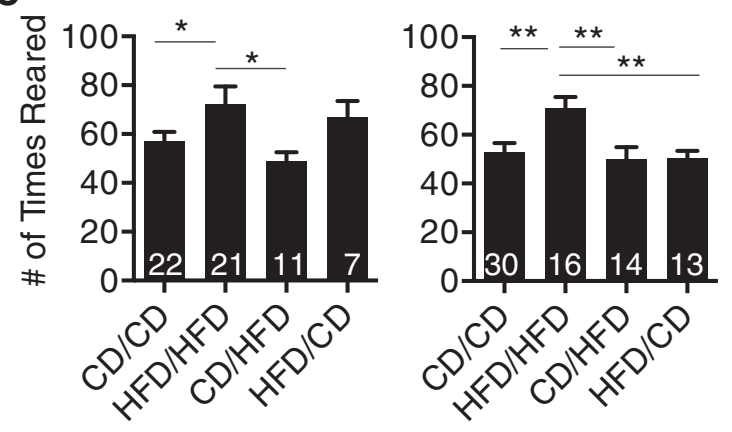

\section{Condition}

Figure 2 Maternal obesity increases anxiety in female offspring with partial amelioration by diet intervention. Male and female offspring from dams fed control diet (CD) or high fat diet (HFD) throughout gestation and lactation or that underwent diet switch at lactation were examined at P32-35 in Open Field Assay (OFA). (A) Mean \pm SEM for distance traveled (one-way ANOVA with post-hoc Fisher's LSD analysis, male $\mathrm{CD} / \mathrm{CD}$ versus HFD/HFD ${ }^{*} P=0.0054, \mathrm{CD} / \mathrm{CD}$ versus $\mathrm{HFD}$ / $C D * P=0.0339$ ). No significant differences were found in female offspring for total distance. (B) Center:total distance ratios for male and female offspring shown as mean \pm SEM (one-way ANOVA with post-hoc Fisher's LSD analysis, female CD/CD versus HFD/HFD $* P=0.0415, C D / C D$ versus $\mathrm{HFD} / \mathrm{CD} * P=0.0362$ ). (C) Number of times reared during OFA shown as mean \pm SEM number for male and female offspring (one-way ANOVA with post-hoc Fisher's LSD analysis, male CD/CD versus HFD/HFD ${ }^{*} P=0.0386, \mathrm{HFD} / \mathrm{HFD}$ versus $\mathrm{CD} / \mathrm{HFD}{ }^{*} P=0.0134$; female $\mathrm{CD} / \mathrm{CD}$ versus HFD/HFD ${ }^{*} P=0.0025, \mathrm{HFD} / \mathrm{HFD}$ versus CD/HFD ${ }^{*} P=0.003, \mathrm{HFD} / \mathrm{HFD}$ versus HFD/CD $\left.{ }^{* *} P=0.005\right)$. $N=7$ to 22 or $N=13$ to 30 per condition for males and females, respectively, as indicated in the graph. Abbreviations: $P$, postnatal day. 


\section{Decreased microglial activation in female offspring from HFD to CD diet switched dams}

Microglia are brain resident immune cells that can modulate the cytokine milieu and impact neuronal function [47-50]. To determine if dietary intervention can reduce maternal HFD-induced microglial activation in offspring, gene expression of $I b a 1$ within brains of male or female offspring from $\mathrm{CD} / \mathrm{CD}$, HFD/HFD, CD/HFD and HFD/CD dams was examined by RT-qPCR. Maternal dietary conditions had no impact on Iba1 gene expression within male offspring. In contrast, within female offspring, a significant increase in Ibal transcript was observed in HFD/HFD relative to $\mathrm{CD} / \mathrm{CD}$ female offspring controls (Figure 4A). Lowering maternal dietary fat content during lactation resulted in a significant decrease in $I b a 1$ transcripts in the brain (HFD/HFD versus HFD/CD). Iba1 immunohistology in the amygdala, a region associated

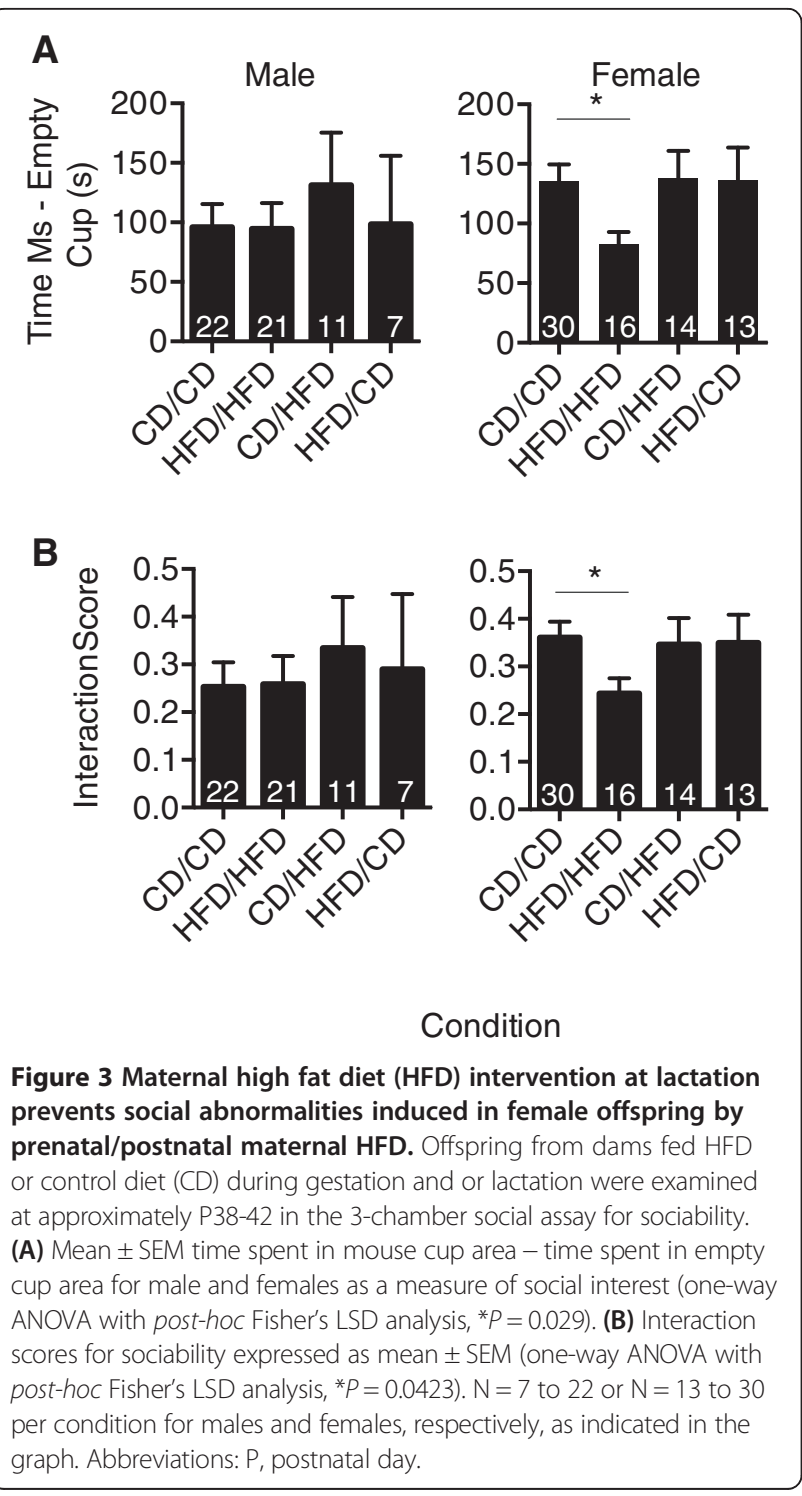

with anxiety and social behaviors, was similar over different maternal dietary conditions in male offspring (Figure 4B,C). However, Iba1 staining revealed that microglia from HFD/HFD female offspring appeared more reactive relative to $\mathrm{CD} / \mathrm{CD}, \mathrm{CD} / \mathrm{HFD}$ and $\mathrm{HFD} / \mathrm{CD}$ female offspring (Figure 4D). Densitometric analysis also showed significantly more Iba1 staining intensity in HFD/HFD female offspring compared to CD/CD controls as well as HFD/ $\mathrm{CD}$ female offspring (Figure 4B). Therefore, maternal HFD likely impacts the microglial population in female offspring and dietary intervention during the lactation period reduces reactivity.

\section{Maternal HFD induces a pro-inflammatory milieu in the brain that is altered by dietary intervention}

To examine whether the pro-inflammatory cytokine milieu corresponded with altered sociability and microglial reactivity patterns, brain lysates of offspring from each group were examined for IL-1 $\beta$ and TNF $\alpha$ levels. No significant differences in either IL- $1 \beta$ or TNF $\alpha$ were detected within male offspring from the different maternal dietary conditions (Figure 5A,B). As was observed with social readouts, female HFD/HFD offspring displayed an elevated level of inflammation in brain tissue (Figure 5A, B). Female HFD/HFD offspring had significantly higher IL- $1 \beta$ and TNF $\alpha$ levels compared to female CD/CD controls. Again, dietary intervention at the point of lactation (that is HFD/CD) resulted in offspring with similar cytokine levels to $\mathrm{CD} / \mathrm{CD}$ dam offspring and, in the case of TNF $\alpha$, was significantly lower than HFD/HFD dam offspring. Together, these data indicate that dietary intervention is able to alleviate the impact of maternal HFD on offspring brain inflammation.

\section{Discussion}

Obesity is rising to staggering proportions in the US [1] and impacts several aspects of human health. While obesity is a comorbidity for several diseases, including diabetes, cancer, and cardiovascular disease [51], studies now also show associations between obesity and altered cognition and behavior [52]. Additionally, maternal HFD and obesity can have significant ramifications in subsequent offspring $[13,14,20,21]$, indicating the importance of pre- and perinatal maternal obesity. It is therefore critical to understand how maternal obesity influences offspring and whether efficacious intervention strategies exist. Here we show that maternal HFD can significantly impact offspring CNS inflammation, hyperactivity, anxiety, and sociability. Critically, we find that maternal dietary intervention during lactation is sufficient to offset many of the maladaptive responses observed in subsequent offspring.

Pre-pregnancy adiposity is also associated with development of ADHD symptoms in children [22-24]. In 


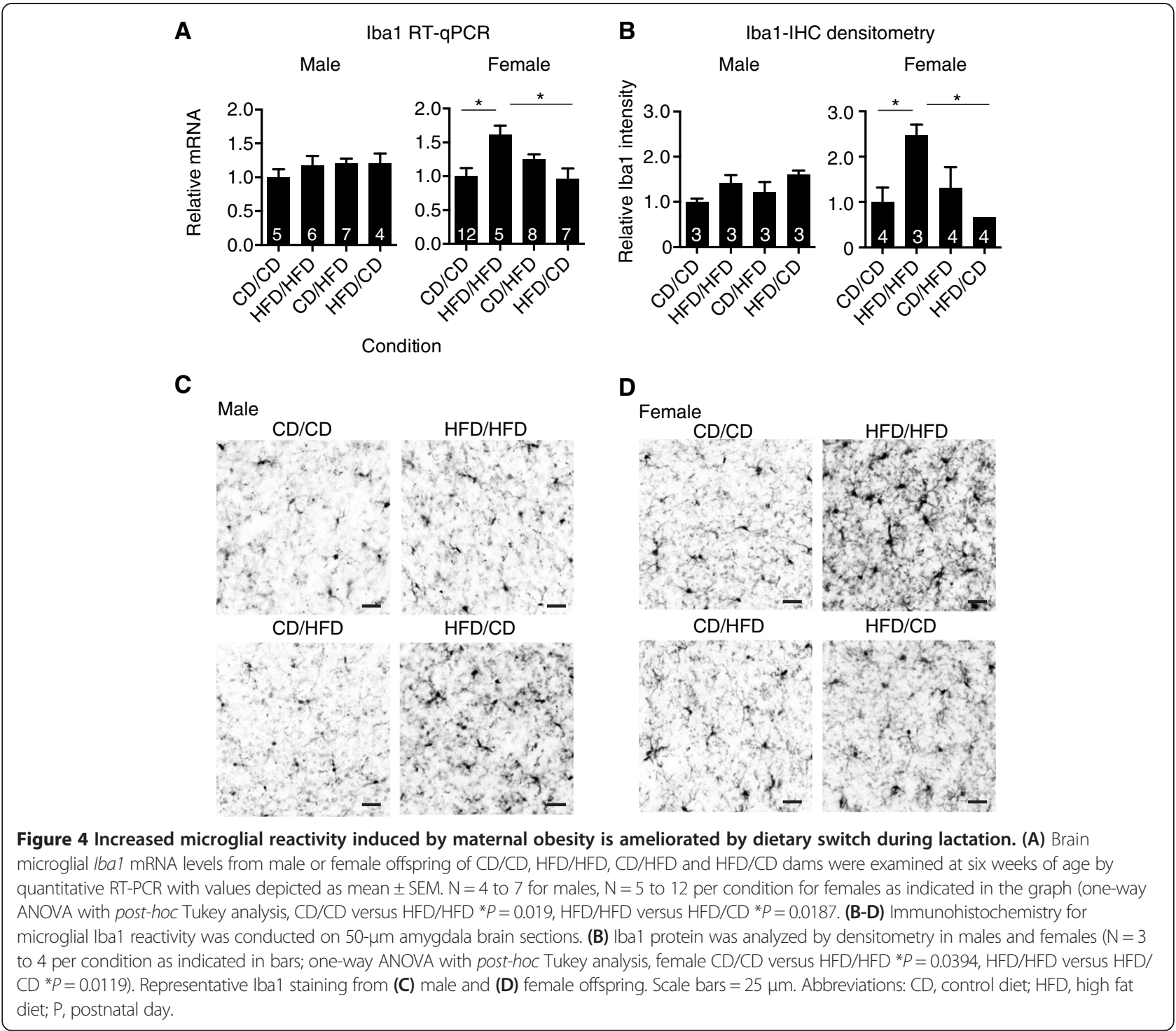

accordance with a previous study [25], we demonstrated that maternal HFD contributes to hyperactivity in subsequent male HFD/HFD and HFD/CD offspring relative to $\mathrm{CD} / \mathrm{CD}$ controls (Figure 2A). Female HFD/HFD offspring showed a similar trend toward increased activity. Maternal HFD feeding during gestation alone was sufficient to induce this response, indicating that this is a sensitive developmental period for hyperactivity phenotypes. Interestingly, increased levels of pro-inflammatory cytokines were not observed across groups in male offspring (Figure 5A,B), suggesting that the hyperactivity phenotype may be associated with a separate parameter. Recently, hyperactivity and impulsivity symptoms in ADHD children have been associated with altered dopaminergic and serotonergic pathways [53] and, in primates, maternal HFD has also been shown to alter offspring serotonergic pathways [19]. This suggests that gestational maternal obesity may alter hyperactivity through long-lasting modifications of the serotonergic pathway.

In other behavioral parameters, we found that maternal HFD during gestation and lactation resulted in significant alterations only within female offspring. In humans, maternal obesity is associated with increased risk of autism (odds ratio $=1.67$ ) [30]. Accordingly, we show that female offspring from HFD/HFD dams had reduced sociability compared to controls (Figure $3 \mathrm{~A}, \mathrm{~B}$ ). Although ASD-like behaviors have been shown in prenatal infection models $[31,32,46]$, our data demonstrate social deficits can occur as a consequence of maternal obesity alone in a rodent model. The interaction score used to assess social interaction normalizes for the total time in both the mouse and empty cup region to more accurately reflect measures of social anxiety rather than a failure to exhibit exploratory behavior. The factors by 


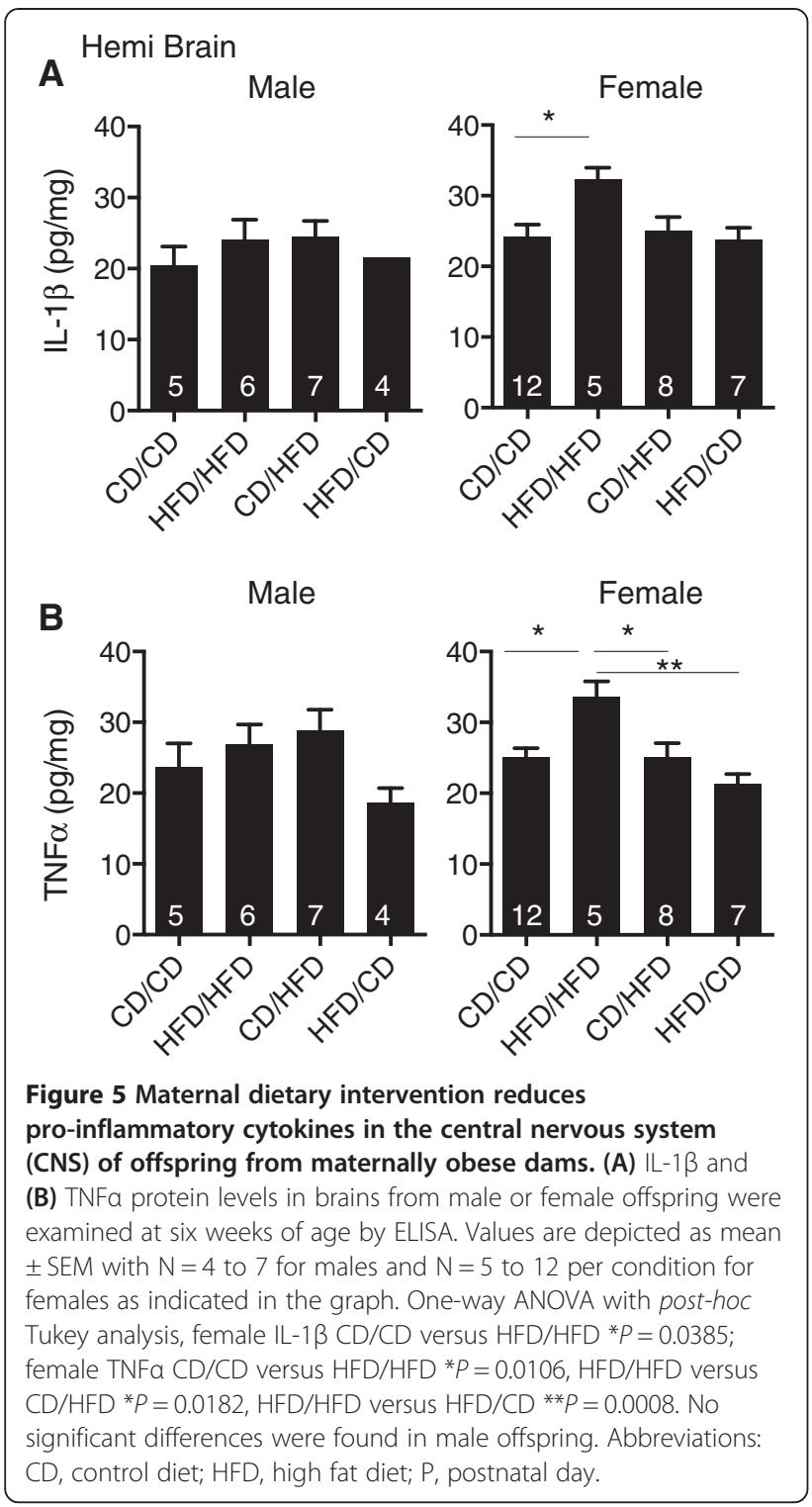

which maternal obesity decreases offspring sociability are unknown; however, obesity-induced low-grade chronic inflammation likely plays a role in altered offspring behavior. Notably, in humans, obese mothers have increased IL-6 in plasma and breast milk and increased IL-6 gene expression in placental macrophages [54-56]. In rodents, increased fetal IL-6 levels from high fat/sugar-fed dams are also observed [57]. Prenatal exposure to IL-6, either through maternal immune activation (MIA) or cytokine injection, is sufficient to cause aberrant ASD-like behaviors and altered brain transcriptomes in offspring [58,59]. Therefore, increased maternal IL-6 likely plays a role in decreased sociability in offspring from obese dams. Although unexplored in our study, maternal obesity also impacts many other aspects of offspring brain homeostasis, such as serotonin pathways, lipid peroxidation, and corticosteroid receptor expression $[12,13,19,21]$ that may also contribute to aberrations in offspring behavior.

In human children and adults, obesity is associated with increased anxiety and associations are sometimes stronger in females [16,57]. Examination of non-human primate offspring from maternally obese mothers also revealed a female bias for elevated anxiety [19]. Likewise, our data demonstrate an increase in anxiety and decreased sociability only within female offspring from HFD/HFD dams (Figure 2B,C). In other rodent models, maternal HFD increased anxiety in both male and female offspring, measured by decreased time spent in the center region of the OFA or the percentage of time in the open arms of elevated plus maze [14,20,21]. Although the reason for discrepancies in affected sex is unclear, in some cases $[14,21]$ this may be a reflection of differences in rat versus mouse models. Previous work has demonstrated developmental sex discrepancies in rat brain microglial colonization [60]. Increased microglial numbers in early postnatal male rat brains may increase susceptibility to early life insults. Currently, it is unknown if mice have similar sex differences in microglial colonization; however, at P3, increased Il1b, Tnfa, and Il6 gene expression has been shown in female brains [61], suggesting that during early mouse development, female brains have a more pro-inflammatory milieu compared to males. This may be important since elevated CNS pro-inflammatory cytokines, including IL-1 $\beta$ and/or TNF $\alpha$, have been associated with increased anxiety, decreased cognition, and altered social behaviors [35-38,62]. Our data demonstrated that elevated brain inflammation corresponded to behavioral manifestations. Significant increases in brain levels of both IL-1 $\beta$ and TNF $\alpha$ were observed only in female offspring from HFD/HFD dams (Figure 5) that were also the only group to display increased anxiety and decreased sociability (Figures 2 and 3). Notably, within male offspring no alterations were observed between groups in regard to neuroinflammation (Figures 4 and 5) or anxiety and sociability parameters (Figures 2 and 3), further supporting the idea of a link between pro-inflammatory cytokines and behavioral deficits in female offspring. Additionally, increased hippocampal IL-1 $\beta$ has been observed in offspring of both sexes with altered behavior [14]. Therefore, regardless of differences in the impacted sex between studies, maternal obesity results in increased offspring CNS inflammation that is associated with behavioral abnormalities.

Microglia are integral resident CNS immune cells that are critical for both homeostatic and pathological states of the CNS [47-50]. Microglia continually survey the brain parenchyma and, upon activation, are capable of rapidly redirecting processes, phagocytosis, and augmenting inflammation via production of reactive oxygen 
species and pro-inflammatory cytokines including IL-1 $\beta$ and TNFo, ([63], 2005; [47,64]). Our data revealed increased brain gene expression and amygdala tissue qualitative and quantitative protein expression of the microglial marker, ionized calcium binding adaptor molecule 1 (Iba1) (Figure 4A,B,D) in female offspring from HFD/HFD dams. This is suggestive of increased microglial activation. However, it is interesting to note that, unlike what was observed within female offspring, altered Iba1 mRNA and protein expression was not observed in male offspring across conditions (Figure 4A,B) that also showed no signs of neuroinflammation (Figure 5A,B). Together with previously reported increased offspring hippocampal Iba1 expression after maternal saturated fat feeding [14], these data suggest that microglial reactivity in multiple brain regions are altered by prenatal/perinatal maternal dietary conditions. Because microglia can selfrenew for CNS maintenance $[47,65]$, early insults may have a long-lived impact. Notably, in perinatal bacterial infection models, microglial Iba1 immunoreactivity was associated with increased anxiety and elevated brain IL-1 $\beta$ levels that corresponded with decreased memory after lipopolysaccharide (LPS) challenge [35,43,66]. Isolated $\mathrm{CD}_{11 \mathrm{~b}^{+}}$microglia from LPS injected mice, previously exposed to perinatal infection, demonstrated increased IL$1 \beta$ secretion, supporting the idea that early exposure to inflammation has long- lasting consequences on microglial reactivity [43]. Although microglia-specific IL-1 $\beta$ and TNF $\alpha$ levels were not specifically examined, maternal obesity likely acts as an early life insult that alters microglial reactivity, allowing them to contribute to elevated offspring brain inflammation.

One of our most important findings is that dietary intervention during lactation alleviates the effects of gestational HFD. Offspring from HFD dams switched to $\mathrm{CD}$ during lactation no longer exhibit aberrations in rearing and social behavior (Figures 2 and 3). Additionally, maternal dietary intervention ameliorated microglia activation and brain pro-inflammatory cytokines observed in offspring of HFD/HFD dams (Figures 4 and 5). An important caveat to note in our study is that rodent and human brain development occurs at different stages with the peaks of gliogenesis and brain growth spurts occurring postnatally in rodents versus prenatally in humans [67]. Therefore, future studies examining diet regiments that are altered later during lactation in rodents, after the majority of glial development is completed, may be more translatable to the human condition. It is likely that early prenatal dietary interventions will have the best efficacy in humans. While the mechanism by which dietary intervention exerts these effects in rodents is unknown, intervention may modulate epigenetic changes caused by maternal diet or impact offspring exposure to maternal pro-inflammatory cytokines or maternal microbiomes.
Alterations in methyl-CpG-binding protein 2 (MeCP2) results in ASD-like behaviors [68-70]. Since MeCP2 binds to methylated DNA, epigenetic changes may alter MeCP2 binding and function. Therefore, it is interesting to note that maternal HFD has been shown to alter methylation in the CNS [71], although it is unknown if it specifically impacts $\mathrm{MeCP} 2$ targets related to ASD-like behaviors. Obesity and HFD significantly shift intestinal microbiota [72-76] and increased BMI is associated with altered, less diverse microbiomes and elevated IL-6 in breast milk [55]. Microbial colonization is thought to occur after birth, making the postnatal period critical for shaping the subsequent microbiome [77]. Interestingly, parental diet has been shown to modulate the microbiome of subsequent offspring [78] and emerging evidence has demonstrated that the microbiome composition can significantly impact immunity, inflammation, and obesity [78-81]. Both chronic stress and autism have been associated with altered microbiomes, and postnatal colonization is implicated in modulation of the hypothalamic-pituitary-adrenal axis suggesting links between brain function and intestinal bacteria [82-84]. Whether dietary intervention is able to shift offspring microbiome to elicit behavioral effects will require further experiments.

In summary, these data demonstrate that maternal obesity results in altered anxiety, hyperactivity, and for the first time, sociability in offspring. Neuroinflammation, reflected by elevated IL- $1 \beta$, TNF $\alpha$, and microglial Iba1 staining and transcripts in affected female offspring, may be a mechanism for the enduring behavioral alterations as a result of pre- and postnatal exposure to maternal HFD. Importantly, our data highlight dietary intervention as a potential therapeutic strategy to offset the deleterious effects of maternal obesity on offspring behavior and CNS inflammation.

\section{Additional files}

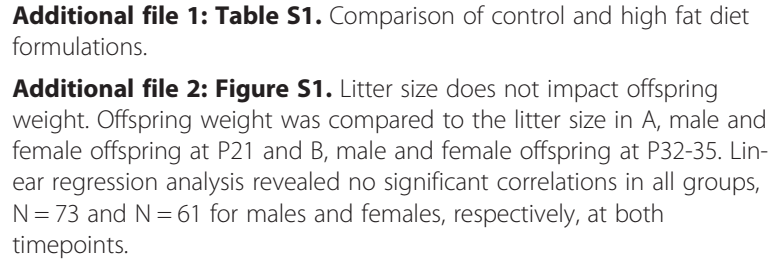

Additional file 1: Table S1. Comparison of control and high fat diet formulations.

Additional file 2: Figure S1. Litter size does not impact offspring weight. Offspring weight was compared to the litter size in A, male and female offspring at P21 and B, male and female offspring at P32-35. Linear regression analysis revealed no significant correlations in all groups, $\mathrm{N}=73$ and $\mathrm{N}=61$ for males and females, respectively, at both timepoints.

\section{Abbreviations}

AHDH: Attention deficit hyperactivity disorder; ANOVA: Analysis of variance; ASD: Autism spectrum disorder; BMI: Body mass index; CNS: Central nervous system; DTT: Dithiothreitol; ELISA: Enzyme-linked immunosorbent assay; HFD: High fat diet; Iba1: Ionized calcium binding adaptor molecule 1; IL: Interleukin; LPS: Lipopolysaccharide; MeCP2: Methyl-CpG-binding protein 2; MIA: Maternal immune activation; NBF: Normal buffered formalin; OFA: Open field assay; P: Postnatal day; PBS: Phosphate buffered saline; PBS-X: phosphate buffered saline with triton- $X ;$ RT: Room temperature; RT-qPCR: real time quantitative polymerase chain reaction; TBSE: Tris buffered saline with EDTA; TNF: Tumor necrosis factor. 


\section{Competing interests}

The authors have no financial competing interest.

\section{Authors' contributions}

SK carried out behavioral, biochemical and histological studies, conducted analysis as well as drafted the manuscript. AK performed behavioral studies. DA and JF assisted in analysis and drafting of the manuscript. All authors read and approved the final manuscript.

\section{Acknowledgements}

We would like to thank Chris Fulcher for technical support. Funding sources for JDF: Mayo Foundation, GHR Foundation, Mayo Clinic Center for Individualized Medicine, Mayo Clinic Gerstner Family Career Development Award. Funding sources for SSK: National Institutes of Health/National Institute of Mental Health (NIH/NIMH) R03 MH103632. Funding sources for DAF: NIH/NIMH R00 MH091238 and R01 MH096773.

\section{Author details}

'Department of Neuroscience, Mayo Clinic Jacksonville, 4500 San Pablo Road, Jacksonville, FL 32224, USA. ${ }^{2}$ Neurobiology of Disease Program, Mayo Graduate School, 200 First Street SW, Rochester, MN 55905, USA. ${ }^{3}$ Department of Behavioral Neuroscience and Psychiatry, Oregon Health and Science University, Portland, OR 97239, USA. ${ }^{4}$ Advanced Imaging Research Center, Oregon Health and Science University, Portland, OR 97239, USA.

Received: 10 April 2014 Accepted: 21 August 2014

Published online: 12 September 2014

\section{References}

1. Flegal KM, Carroll MD, Kit BK, Ogden CL: Prevalence of obesity and trends in the distribution of body mass index among US adults, 1999-2010. JAMA J Am Med Assoc 2012, 307:491-497.

2. Cedergren MI: Maternal morbid obesity and the risk of adverse pregnancy outcome. Obstet Gynecol 2004, 103:219-224.

3. Blomberg M: Maternal obesity, mode of delivery, and neonatal outcome. Obstet Gynecol 2013, 122:50-55.

4. Cnattingius S, Villamor E, Johansson S, Edstedt Bonamy A-K, Persson M, Wikström A-K, Granath F: Maternal obesity and risk of preterm delivery. JAMA J Am Med Assoc 2013, 309:2362-2370.

5. Levin BE, Govek E: Gestational obesity accentuates obesity in obesity-prone progeny. Am J Physiol 1998, 275:R1374-R1379.

6. Shankar K, Harrell A, Liu X, Gilchrist JM, Ronis MJJ, Badger TM: Maternal obesity at conception programs obesity in the offspring. Am J Physiol Regul Integr Comp Physiol 2008, 294:R528-R538.

7. Walker C-D, Naef L, d' Asti E, Long H, Xu Z, Moreau A, Azeddine B: Perinatal maternal fat intake affects metabolism and hippocampal function in the offspring: a potential role for leptin. Ann N Y Acad Sci 2008, 1144:189-202.

8. White CL, Pistell PJ, Purpera MN, Gupta S, Fernandez-Kim S-O, Hise TL, Keller $\mathrm{JN}$, Ingram DK, Morrison CD, Bruce-Keller AJ: Effects of high fat diet on Morris maze performance, oxidative stress, and inflammation in rats: contributions of maternal diet. Neurobiol Dis 2009, 35:3-13.

9. Fraser A, Tilling K, Macdonald-Wallis C, Sattar N, Brion M-J, Benfield L, Ness A, Deanfield J, Hingorani A, Nelson SM, Smith GD, Lawlor DA: Association of maternal weight gain in pregnancy with offspring obesity and metabolic and vascular traits in childhood. Circulation 2010, 121:2557-2564.

10. Odaka Y, Nakano M, Tanaka T, Kaburagi T, Yoshino H, Sato-Mito N, Sato K: The influence of a high-fat dietary environment in the fetal period on postnatal metabolic and immune function. Obes Silver Spring Md 2010, 18:1688-1694.

11. Reynolds RM, Allan KM, Raja EA, Bhattacharya S, McNeill G, Hannaford PC, Sarwar N, Lee AJ, Bhattacharya S, Norman JE: Maternal obesity during pregnancy and premature mortality from cardiovascular event in adult offspring: follow-up of 1,323,275 person years. BMJ 2013, 347:f4539.

12. Tozuka Y, Wada E, Wada K: Diet-induced obesity in female mice leads to peroxidized lipid accumulations and impairment of hippocampal neurogenesis during the early life of their offspring. FASEB J Off Publ Fed Am Soc Exp Biol 2009, 23:1920-1934

13. Tozuka Y, Kumon M, Wada E, Onodera M, Mochizuki H, Wada K: Maternal obesity impairs hippocampal BDNF production and spatial learning performance in young mouse offspring. Neurochem Int 2010, 57:235-247.
14. Bilbo SD, Tsang V: Enduring consequences of maternal obesity for brain inflammation and behavior of offspring. FASEB J Off Publ Fed Am Soc Exp Biol 2010, 24:2104-2115.

15. Desai RA, Manley M, Desai MM, Potenza MN: Gender differences in the association between body mass index and psychopathology. CNS Spectr 2009, 14:372-383.

16. Rofey DL, Kolko RP, losif A-M, Silk JS, Bost JE, Feng W, Szigethy EM, Noll RB, Ryan ND, Dahl RE: A longitudinal study of childhood depression and anxiety in relation to weight gain. Child Psychiatry Hum Dev 2009, 40:517-526.

17. Zhao G, Ford ES, Dhingra S, Li C, Strine TW, Mokdad AH: Depression and anxiety among US adults: associations with body mass index. Int J Obes 2009, 33:257-266.

18. Gariepy G, Nitka D, Schmitz N: The association between obesity and anxiety disorders in the population: a systematic review and meta-analysis. Int J Obes 2010, 34:407-419.

19. Sullivan EL, Grayson B, Takahashi D, Robertson N, Maier A, Bethea CL, Smith MS, Coleman K, Grove KL: Chronic consumption of a high-fat diet during pregnancy causes perturbations in the serotonergic system and increased anxiety-like behavior in nonhuman primate offspring. J Neurosci Off J Soc Neurosci 2010, 30:3826-3830.

20. Peleg-Raibstein D, Luca E, Wolfrum C: Maternal high-fat diet in mice programs emotional behavior in adulthood. Behav Brain Res 2012, 233:398-404.

21. Sasaki A, de Vega WC, St-Cyr S, Pan P, McGowan PO: Perinatal high fat diet alters glucocorticoid signaling and anxiety behavior in adulthood. Neuroscience 2013, 240:1-12.

22. Rodriguez A, Miettunen J, Henriksen TB, Olsen J, Obel C, Taanila A, Ebeling H, Linnet KM, Moilanen I, Järvelin M-R: Maternal adiposity prior to pregnancy is associated with ADHD symptoms in offspring: evidence from three prospective pregnancy cohorts. Int J Obes 2008, 32:550-557.

23. Rodriguez A: Maternal pre-pregnancy obesity and risk for inattention and negative emotionality in children. J Child Psychol Psychiatry 2010, 51:134-143.

24. Van Lieshout RJ: Role of maternal adiposity prior to and during pregnancy in cognitive and psychiatric problems in offspring. Nutr Rev 2013, 71(Suppl 1):S95-S101.

25. Fernandes C, Grayton H, Poston L, Samuelsson A-M, Taylor PD, Collier DA, Rodriguez A: Prenatal exposure to maternal obesity leads to hyperactivity in offspring. Mol Psychiatry 2012, 17:1159-1160.

26. Hinkle SN, Schieve LA, Stein AD, Swan DW, Ramakrishnan U, Sharma AJ: Associations between maternal prepregnancy body mass index and child neurodevelopment at 2 years of age. Int J Obes 2012, 36:1312-1319.

27. Hinkle SN, Sharma AJ, Kim SY, Schieve LA: Maternal prepregnancy weight status and associations with children's development and disabilities at kindergarten. Int J Obes 2013, 37:1344-1351.

28. Atladóttir HO, Thorsen P, Østergaard L, Schendel DE, Lemcke S, Abdallah M, Parner ET: Maternal infection requiring hospitalization during pregnancy and autism spectrum disorders. J Autism Dev Disord 2010, 40:1423-1430.

29. Atladóttir HÓ, Henriksen TB, Schendel DE, Parner ET: Autism after infection, febrile episodes, and antibiotic use during pregnancy: an exploratory study. Pediatrics 2012, 130:e1447-e1454

30. Krakowiak P, Walker CK, Bremer AA, Baker AS, Ozonoff S, Hansen RL, Hertz-Picciotto I: Maternal metabolic conditions and risk for autism and other neurodevelopmental disorders. Pediatrics 2012, 129:e1121-e1128.

31. Patterson $\mathrm{PH}$ : Maternal infection and immune involvement in autism. Trends Mol Med 2011, 17:389-394

32. Malkova NV, Yu CZ, Hsiao EY, Moore MJ, Patterson PH: Maternal immune activation yields offspring displaying mouse versions of the three core symptoms of autism. Brain Behav Immun 2012, 26:607-616.

33. Bilbo SD, Schwarz JM: The immune system and developmental programming of brain and behavior. Front Neuroendocrinol 2012, 33:267-286.

34. Kohman RA, Rhodes JS: Neurogenesis, inflammation and behavior. Brain Behav Immun 2013, 27:22-32.

35. Bilbo SD, Biedenkapp JC, Der-Avakian A, Watkins LR, Rudy JW, Maier SF: Neonatal infection-induced memory impairment after lipopolysaccharide in adulthood is prevented via caspase-1 inhibition. I Neurosci Off J Soc Neurosci 2005, 25:8000-8009.

36. Terrando N, Monaco C, Ma D, Foxwell BMJ, Feldmann M, Maze M: Tumor necrosis factor-alpha triggers a cytokine cascade yielding postoperative cognitive decline. Proc Natl Acad Sci U S A 2010,

107:20518-20522. 
37. Barrientos RM, Hein AM, Frank MG, Watkins LR, Maier SF: Intracisternal interleukin-1 receptor antagonist prevents postoperative cognitive decline and neuroinflammatory response in aged rats. J Neurosci Off J Soc Neurosci 2012, 32:14641-14648.

38. Rossi S, Sacchetti L, Napolitano F, De Chiara V, Motta C, Studer V, Musella A, Barbieri F, Bari M, Bernardi G, Maccarrone M, Usiello A, Centonze D: Interleukin- $1 \beta$ causes anxiety by interacting with the endocannabinoid system. J Neurosci Off J Soc Neurosci 2012, 32:13896-13905.

39. Chez MG, Dowling T, Patel PB, Khanna P, Kominsky M: Elevation of tumor necrosis factor-alpha in cerebrospinal fluid of autistic children. Pediatr Neurol 2007, 36:361-365.

40. Li X, Chauhan A, Sheikh AM, Patil S, Chauhan V, Li X-M, Ji L, Brown T, Malik M: Elevated immune response in the brain of autistic patients. J Neuroimmunol 2009, 207:111-116

41. Depino AM: Peripheral and central inflammation in autism spectrum disorders. Mol Cell Neurosci 2013, 53:69-76.

42. Vargas DL, Nascimbene C, Krishnan C, Zimmerman AW, Pardo CA: Neuroglial activation and neuroinflammation in the brain of patients with autism. Ann Neurol 2005, 57:67-81.

43. Williamson LL, Sholar PW, Mistry RS, Smith SH, Bilbo SD: Microglia and memory: modulation by early-life infection. J Neurosci Off J Soc Neurosci 2011, 31:15511-15521.

44. Beumer W, Gibney SM, Drexhage RC, Pont-Lezica L, Doorduin J, Klein HC, Steiner J, Connor TJ, Harkin A, Versnel MA, Drexhage HA: The immune theory of psychiatric diseases: a key role for activated microglia and circulating monocytes. J Leukoc Biol 2012, 92:959-975.

45. Derecki NC, Cronk JC, Lu Z, Xu E, Abbott SBG, Guyenet PG, Kipnis J: Wild-type microglia arrest pathology in a mouse model of Rett syndrome. Nature 2012, 484:105-109.

46. Shi L, Fatemi SH, Sidwell RW, Patterson PH: Maternal influenza infection causes marked behavioral and pharmacological changes in the offspring. J Neurosci Off J Soc Neurosci 2003, 23:297-302

47. Saijo K, Glass CK: Microglial cell origin and phenotypes in health and disease. Nat Rev Immunol 2011, 11:775-787.

48. Tremblay M-Ė, Stevens B, Sierra A, Wake H, Bessis A, Nimmerjahn A: The role of microglia in the healthy brain. J Neurosci Off J Soc Neurosci 2011, 31:16064-16069.

49. Aguzzi A, Barres BA, Bennett ML: Microglia: scapegoat, saboteur, or something else? Science 2013, 339:156-161.

50. Kettenmann H, Kirchhoff F, Verkhratsky A: Microglia: new roles for the synaptic stripper. Neuron 2013, 77:10-18.

51. Guh DP, Zhang W, Bansback N, Amarsi Z, Birmingham CL, Anis AH: The incidence of co-morbidities related to obesity and overweight: a systematic review and meta-analysis. BMC Public Health 2009, 9:88.

52. Misiak B, Leszek J, Kiejna A: Metabolic syndrome, mild cognitive impairment and Alzheimer's disease - the emerging role of systemic low-grade inflammation and adiposity. Brain Res Bull 2012, 89:144-149.

53. Bralten J, Franke B, Waldman I, Rommelse N, Hartman C, Asherson P, Banaschewski T, Ebstein RP, Gill M, Miranda A, Oades RD, Roeyers H, Rothenberger A, Sergeant JA, Oosterlaan J, Sonuga-Barke E, Steinhausen HC, Faraone SV, Buitelaar JK, Arias-Vásquez A: Candidate genetic pathways for attention-deficit/hyperactivity disorder (ADHD) show association to hyperactive/impulsive symptoms in children with ADHD. J Am Acad Child Adolesc Psychiatry 2013, 52:1204-1212.e1.

54. Challier JC, Basu S, Bintein T, Minium J, Hotmire K, Catalano PM, Hauguel-de Mouzon S: Obesity in pregnancy stimulates macrophage accumulation and inflammation in the placenta. Placenta 2008, 29:274-281.

55. Collado MC, Laitinen K, Salminen S, Isolauri E: Maternal weight and excessive weight gain during pregnancy modify the immunomodulatory potential of breast milk. Pediatr Res 2012, 72:77-85.

56. Farah $\mathrm{N}$, Hogan AE, O'Connor N, Kennelly MM, O'Shea D, Turner MJ: Correlation between maternal inflammatory markers and fetomaternal adiposity. Cytokine 2012, 60:96-99.

57. Desai N, Roman A, Rochelson B, Gupta M, Xue X, Chatterjee PK, Tam Tam H, Metz CN: Maternal metformin treatment decreases fetal inflammation in a rat model of obesity and metabolic syndrome. Am J Obstet Gynecol 2013, 209:136.e1-9.

58. Smith SEP, Li J, Garbett K, Mirnics K, Patterson PH: Maternal immune activation alters fetal brain development through interleukin-6. J Neurosci Off J Soc Neurosci 2007, 27:10695-10702.
59. Hsiao EY, Patterson PH: Activation of the maternal immune system induces endocrine changes in the placenta via IL-6. Brain Behav Immun 2011, 25:604-615.

60. Schwarz JM, Sholar PW, Bilbo SD: Sex differences in microglial colonization of the developing rat brain. J Neurochem 2012, 120:948-963.

61. Crain JM, Nikodemova M, Watters JJ: Microglia express distinct M1 and M2 phenotypic markers in the postnatal and adult central nervous system in male and female mice. J Neurosci Res 2013, 91:1143-1151.

62. Wohleb ES, Fenn AM, Pacenta AM, Powell ND, Sheridan JF, Godbout JP: Peripheral innate immune challenge exaggerated microglia activation, increased the number of inflammatory CNS macrophages, and prolonged social withdrawal in socially defeated mice. Psychoneuroendocrinology 2012, 37:1491-1505.

63. Davalos D, Grutzendler J, Yang G, Kim JV, Zuo Y, Jung S, Littman DR, Dustin ML, Gan W-B: ATP mediates rapid microglial response to local brain injury in vivo. Nat Neurosci 2005, 8:752-758.

64. Nimmerjahn A, Kirchhoff F, Helmchen F: Resting microglial cells are highly dynamic surveillants of brain parenchyma in vivo. Science 2005, 308:1314-1318.

65. Ajami B, Bennett JL, Krieger C, Tetzlaff W, Rossi FMV: Local self-renewal can sustain CNS microglia maintenance and function throughout adult life. Nat Neurosci 2007, 10:1538-1543.

66. Sominsky L, Walker AK, Ong LK, Tynan RJ, Walker FR, Hodgson DM: Increased microglial activation in the rat brain following neonatal exposure to a bacterial mimetic. Behav Brain Res 2012, 226:351-356.

67. Semple BD, Blomgren K, Gimlin K, Ferriero DM, Noble-Haeusslein LJ: Brain development in rodents and humans: identifying benchmarks of maturation and vulnerability to injury across species. Prog Neurobiol 2013, 106-107:1-16.

68. Amir RE, Van den Veyver IB, Wan M, Tran CQ, Francke U, Zoghbi HY: Rett syndrome is caused by mutations in X-linked MECP2, encoding methyl-CpG-binding protein 2. Nat Genet 1999, 23:185-188.

69. Chahrour M, Zoghbi HY: The story of Rett syndrome: from clinic to neurobiology. Neuron 2007, 56:422-437.

70. Yasui DH, Gonzales ML, Aflatooni JO, Crary FK, Hu DJ, Gavino BJ, Golub MS, Vincent JB, Carolyn Schanen N, Olson CO, Rastegar M, Lasalle JM: Mice with an isoform-ablating Mecp2 exon 1 mutation recapitulate the neurologic deficits of Rett syndrome. Hum Mol Genet 2014, 23:2447-2458

71. Vucetic Z, Kimmel J, Totoki K, Hollenbeck E, Reyes TM: Maternal high-fat diet alters methylation and gene expression of dopamine and opioid-related genes. Endocrinology 2010, 151:4756-4764.

72. Ley RE, Bäckhed F, Turnbaugh P, Lozupone CA, Knight RD, Gordon J: Obesity alters gut microbial ecology. Proc Natl Acad Sci U S A 2005 102:11070-11075.

73. Ley RE, Turnbaugh PJ, Klein S, Gordon J: Microbial ecology: human gut microbes associated with obesity. Nature 2006, 444:1022-1023.

74. Turnbaugh PJ, Bäckhed F, Fulton L, Gordon Jl: Diet-induced obesity is linked to marked but reversible alterations in the mouse distal gut microbiome. Cell Host Microbe 2008, 3:213-223.

75. Turnbaugh PJ, Hamady M, Yatsunenko T, Cantarel BL, Duncan A, Ley RE, Sogin ML, Jones WJ, Roe BA, Affourtit JP, Egholm M, Henrissat B, Heath AC, Knight $\mathrm{R}$, Gordon I: A core gut microbiome in obese and lean twins. Nature 2009, 457:480-484.

76. Turnbaugh PJ, Gordon Jl: The core gut microbiome, energy balance and obesity. J Physiol 2009, 587:4153-4158.

77. Rautava S, Luoto R, Salminen S, Isolauri E: Microbial contact during pregnancy, intestinal colonization and human disease. Nat Rev Gastroenterol Hepatol 2012, 9:565-576.

78. Myles IA, Fontecilla NM, Janelsins BM, Vithayathil PJ, Segre JA, Datta SK: Parental dietary fat intake alters offspring microbiome and immunity. J Immunol Baltim Md 2013, 191:3200-3209.

79. Turnbaugh PJ, Ley RE, Mahowald MA, Magrini V, Mardis ER, Gordon Jl: An obesity-associated gut microbiome with increased capacity for energy harvest. Nature 2006, 444:1027-1031.

80. Dimmitt RA, Staley EM, Chuang G, Tanner SM, Soltau TD, Lorenz RG: Role of postnatal acquisition of the intestinal microbiome in the early development of immune function. J Pediatr Gastroenterol Nutr 2010, 51:262-273.

81. Lee YK, Mazmanian SK: Has the microbiota played a critical role in the evolution of the adaptive immune system? Science 2010, 330:1768-1773. 
82. Sudo N, Chida Y, Aiba Y, Sonoda J, Oyama N, Yu X-N, Kubo C, Koga Y: Postnatal microbial colonization programs the hypothalamic-pituitary-adrenal system for stress response in mice. J Physiol 2004, 558:263-275.

83. Cryan JF, Dinan TG: Mind-altering microorganisms: the impact of the gut microbiota on brain and behaviour. Nat Rev Neurosci 2012, 13:701-712.

84. Kang D-W, Park JG, Ilhan ZE, Wallstrom G, Labaer J, Adams JB, Krajmalnik-Brown R: Reduced incidence of Prevotella and other fermenters in intestinal microflora of autistic children. PLoS One 2013, 8:e68322.

doi:10.1186/s12974-014-0156-9

Cite this article as: Kang et al:: Dietary intervention rescues maternal obesity induced behavior deficits and neuroinflammation in offspring. Journal of Neuroinflammation 2014 11:156.

\section{Submit your next manuscript to BioMed Central and take full advantage of:}

- Convenient online submission

- Thorough peer review

- No space constraints or color figure charges

- Immediate publication on acceptance

- Inclusion in PubMed, CAS, Scopus and Google Scholar

- Research which is freely available for redistribution 